\title{
Comparison of expectant vs. excisional/ablative management for CIN2 in the era of HPV testing.
}

\author{
Kevin Tjandraprawira ${ }^{1}$, Adeola Olaitan ${ }^{2}$, Aviva Petrie $^{3}$, Nafisa Wilkinson ${ }^{2}$, and Adam \\ Rosenthal $^{1}$ \\ ${ }^{1}$ University College London EGA Institute for Women's Health \\ ${ }^{2}$ University College London Hospitals NHS Foundation Trust \\ ${ }^{3}$ University College London Eastman Dental Institute
}

October 8, 2020

\begin{abstract}
Objective: To investigate conservative and excisional/ablative treatment outcomes for cervical intraepithelial neoplasia grade 2 (CIN2) following introduction of virological test of cure. Design: Retrospective study of prospectively collected data. Setting: Teaching hospital colposcopy unit. Population: 331 sequential biopsy-proven CIN2 cases. Methods: CIN2 cases diagnosed 01/07/2014-31/12/2017 were followed-up until colposcopy discharge and then using the national cervical cytology database. Outcomes were defined: Cytological/histological regression was absence of high-grade CIN on biopsy and/or high-grade dysplasia; Virological regression was cytological/histological regression and negative human papillomavirus testing; Persistence was biopsy-proven CIN2 and/or moderate dyskaryosis; Progression was biopsy-proven CIN3+ and/or severe dyskaryosis. Main outcome measures: Regression, persistence, progression rates; median regression/progression times; referral to discharge interval; subsequent CIN. Results: Median follow-up was 22.6 months (range: 1.9-65.1). 175 (52.9\%) patients were initially managed conservatively. $77.3 \%(133 / 172)$ regressed, $13.4 \%(23 / 172)$ persisted and 9.3\%(16/172) progressed to CIN3+. 97(56.4\%) patients achieved virological regression. Median regression and progression times were 6.1 (range 2.4-30.4) months and 7.6 (range 3.843.3) months, respectively. $156(47.1 \%)$ patients underwent initial excision/ablation, with a 89.4\%(110/123) virological cure rate. Patients managed conservatively vs. planned excision spent a median of 16.4 and 11.7 months respectively, within colposcopy follow-up. 7 (4.0\%) and $3(1.9 \%)$ patients developed further CIN in the conservative and treatment groups respectively, during median 17.2 months post-discharge. Conclusions: Conservative management is a reasonable and effective management strategy in appropriately-selected women with CIN2. The above data provide useful information for clinicians and patients deciding management options. Funding: none Keywords: CIN2; conservative; management; expectant; regression; progression; persistence; excision; HPV; virological
\end{abstract}

\section{Introduction}

Cervical intraepithelial neoplasia (CIN) is a human papillomavirus (HPV)-induced precursor to cervical cancer.(1) In the UK, most CIN patients harbor high-risk HPV (HR-HPV) types 16 and 18.(2,3) Up to 80\% of CIN2 cases are infected with high-risk HPV, with types 16, 31 and 52 being most frequently encountered.(4) In addition to the prerequisite HPV infections, there are other risk-factors for CIN, including smoking, immunosuppression, multiple sexual partners, multiparity, long-term oral contraceptive use and other sexually transmitted infections. (5-7)

Most HR-HPV infections only cause low-grade cervical disease and $>90 \%$ resolve within 2 years. $(1,8,9)$ This is due to a successful immune response against HPV.(9,10) However, persistent HPV infections can lead to cervical cancer.(1, 11) This is mediated through unchecked activity of HR-HPV E6 and E7 oncoproteins, allowing the accumulation of mutations leading to carcinogenesis. (11) 
CIN2's natural history is the least described of the 3 grades of CIN (CIN1,2,3).(12) It may regress to CIN1/no CIN, when there is a successful immune response directed toward the HPV proteins, particularly E2 and E6. $(9,10,12,13)$ However, failed HPV eradication leads to CIN2 persistence/progression.(14)

Current UK guideline recommends excisional treatment for CIN2 and 3.(15) Large loop excision of the transformation zone (LLETZ) is the commonest method. $(15,16)$ After treatment, test of cure (TOC) is carried out.(15,17) Negative cytology and HPV results on TOC are reassuring and confer $<1 \%$ risk of CIN2 development in the next 5 years.(18) However, excisional treatment is linked to increased future risks of adverse obstetric and gynaecologic outcomes, e.g. premature labor, preterm delivery and cervical stenosis. $(16,19,20)$

Conservative management is increasingly favored for CIN2.(21) It consists of cytology and colposcopy every 4-6 months.(22) As it spares the women excisional treatment, it is particularly attractive among young, nulliparous patients.(22) However, data on the efficacy of conservative management, when assessed by virological as well as cytological/histological parameters have not previously been published. This study aims to investigate the outcomes of conservative management of CIN2 patients at a teaching hospital (University College London Hospital; UCLH) colposcopy unit, and where possible, compare these with the efficacy of excisional/ablative treatment. Crucially, the study incorporates HPV testing in addition to routine cytological, and where indicated, histological follow-up.

\section{Methods}

This is a retrospective study using prospectively collected data on women with biopsy-proven CIN2. These patients were referred by the national cervical screening program (NHSCSP), their GPs, or via internal referral. All received their first CIN2 diagnoses between 01/07/2014-31/12/2017. Exclusion criteria were:

1. Biopsy reports unable to confirm definite CIN2.

2. Earlier biopsies more severe than CIN2 (CIN3+).

3. Receiving first CIN2 diagnosis prior to the recruitment period.

4. Receiving first CIN2 diagnosis at treatment (either 'see and treat' or planned following prolonged low-grade cytology/biopsy/CIN1).

5. Lost to follow-up after first colposcopy appointment at which CIN2 was diagnosed on biopsy.

6. Patients whose histopathology slides from elsewhere were sent to UCLH for review only and patient was managed at referring hospital.

Two UCLH databases, Clinical Data Repository (UCLH in-house) and CompuScope (Iris-soft, Sale, UK) were utilized to collect data: patient demographics, cervical cytology, colposcopic impression, biopsy, smear and HPV tests. For patients treated with excision, additional data on procedure details, excisional margins and TOC were collected. Follow-up continued until 12 July 2019. UCLH had access then to stand-alone HPV testing cervical swabs (Hybrid Capture, Digene Corporation, Gaithersburg, MD), in addition to standard liquid-based cytology (LBC) HPV testing for TOC (Abbott RealTime High Risk HPV Assay, Abbott Laboratories, Abbott Park, Illinois, USA) as part of the NHSCSP.

The initial conservative management group was routinely followed up every 6 months and underwent cervical LBC sampling, stand-alone HR-HPV testing and colposcopy with or without biopsy. Follow-up visits were categorised as follows:

Regression (cytological/histological): no CIN, or CIN1-only on cervical biopsy; and/or negative cytology or mild dyskaryosis or borderline nuclear changes on LBC.

Virological regression $=$ cytological regression and negative HPV testing.

1. Persistence: CIN2 on cervical biopsy and/or moderate dyskaryosis on LBC.

2. Progression: CIN3 or invasive cervical cancer on subsequent cervical biopsy and/or severe dyskaryosis or suspected invasion on LBC. Progression was not based on cervical colposcopic impression. 
If the patient's lesion had apparently initially improved but then reverted to high-grade CIN whilst still under colposcopy follow-up, the worst diagnosis was used for the outcome. Patients who remained 'regressed' continued on conservative management until discharge. Patients with cytological/histological evidence of persistent CIN2 either continued on conservative management or underwent excision, based on clinician's discretion and/or patient request. Patients progressing to CIN3+ were recommended to undergo excision. These women were then followed up using the in-house databases (as above) to identify those who were rereferred to colposcopy. Those not re-referred to UCLH were followed-up via the NHS Open Exeter national cervical cytology database to check their subsequent cytology results.

The initial excision/ablation group were those advised to undergo the procedure following initial colposcopy appointment. Whilst LLETZ was the commonest treatment modality, diathermy ablation and cold knife conization $(\mathrm{CKC})$ were also available according to individual patient circumstances. After treatment, patients were invited for TOC at 6 months, consisting of a repeat cytology and HPV test (performed on the LBC sample). In accordance with national guidance, patients were discharged if their TOC was negative for HR-HPV (15), or invited for additional follow-up if their TOC result was HPV-positive, or if their cytology was high-grade.

All standard haematoxylin and eosin histopathology slides were assessed by UCLH's team of specialist gynaecological pathologists. The slides were assessed for histological diagnosis, and depth of excision and excisional margins (involved or not involved), where appropriate. Overall margin status was 'not involved' if all reported margins were not involved; otherwise, the overall status was 'involved'. p16 immunohistochemical staining was not routinely used.

Patients were allocated outcomes as above according to their status at the end of colposcopy follow-up. For those still under follow-up and for outcomes subsequent to discharge, data was censored on 12 July 2019. Successful conservative management (i.e. 'cure') was defined as no CIN on biopsy; and/or negative or borderline/mild cytology on at least 1 subsequent visit, with no evidence of subsequent high-grade disease whilst still in colposcopy follow-up. Successful excisional/ablative treatment was defined as negative/borderline/mild cytology andHPV-negative on their TOC, or on at least 1 subsequent visit should their initial TOC fail. Patients not attending any of their appointments were classified as 'non-compliant'.

Finally, the NHS OpenExeter cervical cytology database was used to collect data on smears performed outside colposcopy for both routine recall and noncompliant patients. Any records of subsequent abnormal cytology were recorded and classified as indicating a new episode of CIN. Patients who did not have any further smear reports on OpenExeter before or after their expected next smear date were classified as 'lost to follow-up'.

The following outcomes were also assessed:

- Time to regression (calculated from the date of CIN2 diagnosis until the first date of CIN2 regression)

- Time to progression (calculated from the date of CIN2 diagnosis until the first date of CIN2 progression)

- Interval between referral and discharge (calculated from referral date until discharging appointment date)

- Number of visits required (starting from the first colposcopy appointment at which CIN2 was diagnosed until discharge).

- Follow-up duration (assessed from the date of first CIN2 diagnosis until the last known appointment from UCLH and/or OpenExeter database, whichever was the later).

The UK Health Research Authority Application system confirmed that formal ethical review of this audit of routine clinical practice in our institution was not required. There was no patient involvement in this study. This study received no funding. The senior author (ANR) was supported by the NIHR Biomedical Research Centre at University College London Hospitals National Health Service Foundation Trust and University College London.

Statistical Methods 
Descriptive statistics were used for both categorical and continuous variables where appropriate. Analytic statistics were also performed; continuous variables were compared using the independent samples Student's $t$-test, Mann-Whitney U-test or Kruskal-Wallis test, as appropriate. Categorical variables were analyzed by Fisher's exact test or the chi-squared test, depending on minimum samples sizes requirements. Because patients were followed-up for different lengths of time following diagnosis, a Cox proportional hazard regression analysis was performed and survival curves were produced. A significance level of 0.01 was used throughout to account for multiple testing.

Data was entered into Microsoft? Excel for Mac v16.16.3 (Microsoft?, Redmond, USA) and analysed using Statistical Product and Service Solutions SPSS for Mac v.23 (IBM Corp, Armonk, New York, USA) and Stata (StataCorp. 2019. Stata Statistical Software: Release 16 . College Station, TX: StataCorp LLC..

\section{Results}

The CONSORT (Consolidated Standards of Reporting Trials) diagram (Fig. 1) describes patients flow. Initially, 563 patients were identified from pathology reports. 232 were excluded for the reasons shown. 331 patients were eligible for inclusion, of which 175 (52.9\%) were in the initial conservative management group and $156(47.1 \%)$ in the initial planned excisional/ablative treatment group.

The overall median follow-up was 22.6 months (range 1.9-65.1 months). Table 1 compares the conservative vs. initial treatment groups.Follow-up time did not differ between the two groups $(p=0.651)$. Patients initially managed conservatively were significantly younger than patients in the planned treatment group (median 27 vs. $28 \mathrm{yr}, \mathrm{p}=0.032$ ), and had significantly fewer high-grade referral smears than patients in the planned treatment group (43/174 (24.7\%) vs. 88/155 (56.8\%), p<0.001).

Patients in the conservatively managed group had significantly fewer high-grade colposcopic impressions of their cervix compared with the planned treatment group $(37 / 173(21.4 \%)$ vs. $89 / 156(57.1 \%), \mathrm{p}<0.001)$.

Conservatively managed patients had more 'CIN1-2' rather than 'CIN2' on biopsy $(58.3 \%(102 / 175)$ vs. $41.7 \%(73 / 175)$, compared with the planned treatment group $(26.3 \%$ (41/156) vs. $73.7 \%$ $(115 / 156))(\mathrm{p}<0.001)$.

\section{Conservative Management Group (Table 2)}

175 patients were initially managed conservatively; 3 were transferred to other hospitals. Of the remaining 172 patients, $133(77.3 \%)$ underwent cytological/histological regression and 97 (56.4\%) achieved virological regression. $23(13.4 \%)$ patients persisted as CIN2 and $16(9.3 \%)$ progressed to CIN3. There was no statistically significant age difference among patients regressing, persisting and progressing $(\mathrm{p}=0.135)$.

The median regression and progression times were 6.1 months (range 2.4-30.4 months) and 7.6 months (range 3.8-43.3 months), respectively. Cox regression analysis indicated that the regression time was neither influenced by smoking status (HR 1.61 (95\% CI 1.06-2.44), p=0.025) nor parity (HR 0.86 (95\%CI 0.51-1.43), $\mathrm{p}=0.557)$.

There was no evidence that progression time was influenced by smoking (HR 3.87, 95\%CI (0.95-15.88), $\mathrm{p}=0.060$ ) or parity (HR 1.93, 95\% CI (0.53-7.09), $\mathrm{p}=0.321)$. The median interval between referral and discharge was 16.4 months (range 3.9-51.3 months) and the interval was neither significantly influenced by smoking (HR 1.31 (95\%CI 0.13-12.83), $\mathrm{p}=0.818$ ) nor parity (HR 2.07 (95\%CI 0.21-20.4), $\mathrm{p}=0.534$ ) as assessed by Cox regression.

Among patients initially managed conservatively, 36 (20.9\%) subsequently underwent LLETZ; 11 (30.6\%) of these had CIN3 in their specimen. Post-treatment, all patients were invited for TOC. 32 (88.9\%) patients attended and $20(62.5 \%)$ of these had a negative result. Following further follow-up, $23(71.9 \%)$ patients had received a negative TOC at censoring. The median time between treatment and negative TOC was 6 months (range 3-38 months).

\section{Excisional/Ablative Treatment Group (Table 3)}


156 patients were in the initial planned excisional/ablative treatment group. 144 (92.3\%) patients underwent LLETZ, $5(3.2 \%)$ patients underwent CKC, $6(3.8 \%)$ patients underwent diathermy ablation and $1(0.7 \%)$ patient was transferred to another hospital. Of patients undergoing excision (LLETZ/CKC), 93 (64.6\%) had uninvolved margins. 6 months post-treatment, the apparent cure rate was $73.2 \%$. Following further followup (median 21.3 months, range 2.9-65.1 months) and 2 patients undergoing repeat LLETZ, the apparent cure rate for all patients undergoing planned treatment (LLETZ, CKC and diathermy ablation) was 89.4\% at censoring. The apparent cure rate was significantly higher in patients aged [?]30 years old $(\mathrm{p}<0.001)$ and in non-smokers $(\mathrm{p}=0.003)$. There was no significant influence on the apparent cure rate by patient age (HR 0.82 (95\%CI 0.50-1.34), $\mathrm{p}=0.421)$, smoking (HR 0.80 (95\%CI 0.50-1.29), $\mathrm{p}=0.358)$, parity (HR $0.89(95 \% \mathrm{CI}$ 0.49-1.59), $\mathrm{p}=0.684$ ), endocervical margin (HR 0.63 (95\% CI 0.14-2.84), $\mathrm{p}=0.543)$, ectocervical margin (HR 2.49 (95\% CI 0.66-9.39), $\mathrm{p}=0.177$ ), radial margin (HR 0.87 (95\% CI 0.43-1.73), $\mathrm{p}=0.685$ ) or overall margin status (HR 0.56 (95\% CI (0.15-2.06), $\mathrm{p}=0.381)$.

Of the initial planned treatment group, the median interval between referral to discharge was 11.7 months (range: 2.9-74.8 months), and did not differ significantly from that in the conservatively managed group (median 16.4, range 3.9-51.3 months) ( $\mathrm{p}=0.136)$, nor did the number of visits required prior to discharge (median 3 (range: 2-8) in the immediate treatment group vs. median 3 (range: 2-8) in the conservative group, $\mathrm{p}=0.179)$.

\section{Subsequent Disease Development}

$114(60 \%)$ of women due their next smear following discharge within the study follow-up period had a smear report on Open Exeter. 10(3\%) patients had evidence of subsequent disease; 7(70\%) from the conservative management group and $3(30 \%)$ from the excisional group $(\mathrm{p}=0.185)$. Overall, the median interval to subsequent dyskaryosis was 17.2 months (range: $0.2-43.9$ months). The time to development of subsequent dyskaryosis did not differ significantly (median 16.9 vs. 23.4 months in the conservative vs. treatment groups respectively, $\mathrm{p}=0.267$ ). Among conservatively managed patients, smoking (HR 1.31 (95\%CI 0.133$12.83, \mathrm{p}=0.818)$ and parity (HR $2.07(95 \% \mathrm{CI} 0.21-20.40), \mathrm{p}=0.534)$ did not significantly influence disease recurrence. To date, no patients in the conservative management group have developed cervical cancer. Among patients undergoing initial excision, none of the following factors (age at referral (HR $0.70(95 \% \mathrm{CI}$ 0.02-22.84), $\mathrm{p}=0.840$ ), smoking (HR 0.35 (95\%CI 0.02-6.90), $\mathrm{p}=0.490)$, parity (HR 3.88 (95\%CI 0.22-68.33), $\mathrm{p}=0.354$ ), overall margin status (HR -, $\mathrm{p}[?] 0.999)$ significantly predicted disease recurrence.

\section{Discussion}

\section{Main Findings}

Out of 172 patients managed conservatively, 133 (77.3\%) patients underwent cytological/histological regression but the histological and virological regression rates were not significantly higher among patients [?]30 years old $(\mathrm{p}=0.84$ and $\mathrm{p}=0.86$, respectively). This differs from a previous meta-analysis, in which regression increased to $50 \%$ over 24 months and was higher among women $<30$ years old.(12) However, there is little consistency in the definitions of regression, persistence and progression in the papers cited. Our $9.3 \%$ progression rate at 60 months was lower than that in the meta-analysis, which increased from $14 \%$ at 12 months to $24 \%$ at 36 months.(12)

Unsurprisingly, the virological regression rate $(56.4 \%)$ was lower than the cytological/histological regression rate $(77.3 \%)$. This likely reflects persistent HPV infection despite apparent CIN resolution. Such differences should be considered when designing future studies of conservative management.

9 patients in the conservative management group had been referred with severe dyskaryosis. Generally, these would be selected for treatment. However, low-grade colposcopy impressions and absence of CIN3 on biopsy were among the reasons they initially received conservative management. While 4 (44.5\%) of these 9 patients eventually underwent LLETZ, the rest regressed histologically and were discharged to routine recall. Hence, severe dyskaryosis at referral should not immediately preclude patients from conservative management.

Following planned initial treatment, younger age ([?]30 years old) and non-smoking status significantly 
increased our cure rates $(\mathrm{p}<0.001$ and $\mathrm{p}=0.003$, respectively). The link between age and cure rate has been previously reported(23), as has the link between non-smoking and cure; smokers had thrice greater odds of treatment failure than non-smokers. $(24)$

We observed a higher disease recurrence risk in conservatively-managed patients than in those initially undergoing treatment ( $70 \%$ vs. $30 \%, \mathrm{p}=0.185$ ). According to Wilkinson et al, despite having spontaneously regressed, conservatively managed patients remain at increased risk for high-grade disease for at least 5 years.(17)

\section{Strengths and Limitation}

This study took place within the highly-regulated UK national cervical screening program, but with the additional benefit of access to stand-alone HPV testing enabling us to evaluate virological regression in addition to cytological/histological regression. To our knowledge, this is the UK's largest reported CIN2 cohort, the $6^{\text {th }}$ largest reported worldwide and crucially, the first to report on virological regression rates. Finally, our study adds to the limited published data comparing the outcomes of conservative and excisional/ablative treatments for CIN2 in the same cohort, of which ours is the only such study to have investigated virological regression. $(13,17,22,25-29)$

The study has several limitations. $21.4 \%$ of included patients (70/331) were noncompliant with and/or lost to follow-up, and this in combination with our median follow-up of 22.6 months, means that we may have under-estimated the true disease recurrence rate. There was incomplete information on some risk factors, such as smoking status. Despite this, smoking was still found to be significantly inversely associated with successful TOC. The clinical HPV assays used for testing differed in the post-treatment tests done as part of the national program vs. those done as stand-alone tests in the conservatively-managed group; although this was unlikely to introduce significant bias, given the equivalence of the tests used.(30) The study's retrospective nature made it hard to pinpoint the exact reason why some patients were offered conservative management rather than initial treatment. In addition, planned follow-up schedules of the conservatively managed group, and decisions to undergo subsequent treatment were down to clinician/patient preferences, rather than a clinical trial protocol. Also, this audit of the outcomes of routine clinical protocol at our hospital may not be generalizable to other healthcare settings. Finally, the absent of routine p16 immunostaining in equivocal cases of CIN2 means we cannot be certain that all the 'CIN2' cases were high-grade according to the Lower Anogenital Squamous Terminology (LAST) criteria.(31)

It also became apparent that no standard definition of CIN2 regression, persistence and progression has been adopted. Whilst not a limitation to this study, it prevented us from accurately comparing our results with other studies. An international consensus on defining regression, persistence and progression would be helpful, but would be difficult in countries which do not routinely differentiate between CIN2 and CIN3 when reporting high-grade cervical samples.

Furthermore, we could not compare the two groups with respect to regression, persistence and progression as these variable could not be measured in the planned treatment group due to the inherent nature of this management method.

\section{Interpretation}

The planned treatment group presented with evidence of more severe disease (e.g. high-grade smears) compared to the conservatively-managed group.(25) Furthermore, initially treated patients had significantly more high-grade colposcopic impressions $(\mathrm{p}<0.001)$ and more 'CIN2 alone' on biopsy $(\mathrm{p}<0.001)$ compared with the conservatively-managed group. These histological findings have been previously reported. (22)

Patients in the planned treatment group were not significantly older than conservatively managed patients, but there was a trend. This may have been a reflection of them being more likely to have true high-grade disease (as evidence by more 'CIN2 only' rather than 'CIN1-2' biopsies), and/or being more likely to be parous. 
Our regression rate was $77.3 \%$ while another London hospital's cohort was $57 \%$.(26) This difference might be explained by the different regression criteria in the latter study (negative cytology on 2 consecutive visits and no high-grade colposcopic impression).(26)

We compared our results to all available similar studies from the last 10 years. A number of issues arose; some focused exclusively on patients $<25$ years old $(13,17,22,27,28,32)$, and some were laxer in their CIN2 inclusion policy, i.e. inclusion of CIN2-3 as CIN2.(25,33) Whilst many had adopted a similar regression definition to our own, "conservative management success" was less consistently-defined.(17,25) Some were strict on requiring a certain number of consecutive negative cytology results, but others lacked such a definition. $(17,25,29,33)$ Studies varied on defining "persistence" and "progression". $(13,22,27,28)$ Some included "CIN2-3" as persistent, rather than progressive disease. $(13,22,27,28)$ Some defined progression exclusively as invasive cervical cancer. $(13,28)$

With no uniform definitions, we could not compare our results directly with other studies. However, most had adopted broadly similar cytological regression definition and reported high regression rates. This, along with our results, suggests that conservative management is justified in appropriately-selected CIN2 patients. This has recently been suggested by a Danish study involving $>12,000$ CIN2 patients managed conservatively.(34)

Our post-treatment TOC cure rates were high and positively influenced by the patients' young age. This might reflect better HPV clearance in younger women, and/or lower disease severity compared to older women.(12)

Two patients in the initial treatment group required repeat LLETZ due to persistent high-grade disease. In 1 patient whose margins were clear, this would likely have been due to HR-HPV persistence and/or reinfection despite apparently successful excision.(18) Post-treatment HPV status is known to predict future cervical disease development. (23)

Patients managed conservatively developed subsequent disease sooner. This was not statistically significant, possibly due to the low number of patients with sequent disease ( 7 and 3 in the planned conservative and treatment groups, respectively), which in itself was reassuring.

A previous meta-analysis found that involved margins increase the chances of residual and/or recurrent CIN.(35) We did not observe this in our cohort, possibly because of low numbers with recurrent disease and/or our relatively short follow-up.

\section{Conclusion}

Conservative management consisting of regular colposcopy follow-up, combined with biopsy, cervical smear and HPV testing is a reasonable and effective management strategy for appropriately-selected patients with CIN2. Furthermore, our data provide useful information for clinicians and patients when deciding management options in the era of HPV testing. Randomized controlled trials operating under clear protocols with standard definitions of regression, persistence and progression, and longer follow-up periods are required to determine the optimum approach for conservative management in these patients.

\section{Disclosure of Interests}

The authors declare that we do not have any conflicts of interest.

\section{Contribution to Authorship}

ANR and KDT conceived and designed the study. KDT acquired the data. KDT and AP analysed the data. KDT and AR interpreted the data and drafted the manuscript. NW contributed to the acquisition of data. AAO contributed to data interpretation. All authors contributed to the drafting of the article and approved the final version to be published. All authors agree to be accountable for all aspects of the work.

\section{Details of Ethics Approval}


The UK Health Research Authority Application system confirmed that formal ethical review of this audit of routine clinical practice in our institution was not required.

\section{Funding}

This study received no funding.

\section{Reference:}

1. de Sanjosé S, Brotons M, Pavón MA. The natural history of human papillomavirus infection. Best Practice \& Research Clinical Obstetrics \& Gynaecology. 2018;47:2-13.

2. Howell-Jones R, Bailey A, Beddows S, Sargent A, de Silva N, Wilson G, et al. Multi-site study of HPV type-specific prevalence in women with cervical cancer, intraepithelial neoplasia and normal cytology, in England. Br J Cancer. 2010;103(2):209-16.

3. Anderson LA, O'Rorke MA, Wilson R, Jamison J, Gavin AT. HPV prevalence and type-distribution in cervical cancer and premalignant lesions of the cervix: A population-based study from Northern Ireland. J Med Virol. 2016;88(7):1262-70.

4. Bello BD, Spinillo A, Alberizzi P, Cesari S, Gardella B, D'Ambrosio G, et al. Cervical infections by multiple human papillomavirus (HPV) genotypes: Prevalence and impact on the risk of precancerous epithelial lesions. J Med Virol. 2009;81(4):703-12.

5. Roura E, Castellsague X, Pawlita M, Travier N, Waterboer T, Margall N, et al. Smoking as a major risk factor for cervical cancer and pre-cancer: results from the EPIC cohort. Int J Cancer. 2014;135(2):453-66.

6. Chelimo C, Wouldes TA, Cameron LD, Elwood JM. Risk factors for and prevention of human papillomaviruses (HPV), genital warts and cervical cancer. J Infect. 2013;66(3):207-17.

7. Dugue PA, Rebolj M, Garred P, Lynge E. Immunosuppression and risk of cervical cancer. Expert Rev Anticancer Ther. 2013;13(1):29-42.

8. Winer RL, Hughes JP, Feng Q, Xi LF, Cherne S, O'Reilly S, et al. Early natural history of incident, type-specific human papillomavirus infections in newly sexually active young women. Cancer Epidemiol Biomarkers Prev. 2011;20(4):699-707.

9. Stanley MA. Epithelial Cell Responses to Infection with Human Papillomavirus. Clin Microbiol Rev. 2012;25(2):215-22.

10. Stanley M. HPV - immune response to infection and vaccination. Infect Agent Cancer. 2010;5:19-.

11. zur Hausen H. Papillomaviruses and cancer: from basic studies to clinical application. Nat Rev Cancer. $2002 ; 2(5): 342-50$.

12. Tainio K, Athanasiou A, Tikkinen KAO, Aaltonen R, Cárdenas J, Hernándes, et al. Clinical course of untreated cervical intraepithelial neoplasia grade 2 under active surveillance: systematic review and metaanalysis. BMJ. 2018;360:k499.

13. Loopik DL, Bekkers RLM, Massuger LFAG, Melchers WJG, Siebers AG, Bentley J. Justifying conservative management of CIN2 in women younger than 25 years - A population-based study. Gynecol Oncol. 2019;152(1):82-6.

14. McBride AA, Warburton A. The role of integration in oncogenic progression of HPV-associated cancers. PLoS Pathog. 2017;13(4):e1006211.

15. England PH. NHS Cervical Screening Program - Colposcopy and Programme Management. London: Public Health England; 2016.

16. Petry KU. Management options for cervical intraepithelial neoplasia. Best Practice \& Research Clinical Obstetrics \& Gynaecology. 2011;25(5):641-51. 
17. Wilkinson TM, Sykes PH, Simcock B, Petrich S. Recurrence of high-grade cervical abnormalities following conservative management of cervical intraepithelial neoplasia grade 2. Am J Obstet Gynecol. 2015;212(6):769.e1-7.

18. Gosvig CF, Huusom LD, Andersen KK, Duun-Henriksen AK, Frederiksen K, Iftner A, et al. Long-term follow-up of the risk for cervical intraepithelial neoplasia grade 2 or worse in HPV-negative women after conization. Int J Cancer. 2015;137(12):2927-33.

19. Sadler L, Saftlas A, Wang W, Exeter M, Whittaker J, McCowan L. Treatment for cervical intraepithelial neoplasia and risk of preterm delivery. JAMA. 2004;291(17):2100-6.

20. Baldauf JJ, Dreyfus M, Ritter J, Meyer P, Philippe E. Risk of cervical stenosis after large loop excision or laser conization. Obstet Gynecol. 1996;88(6):933-8.

21. Macdonald M, Smith JHF, Tidy JA, Palmer JE. Conservative management of CIN2: National Audit of British Society for Colposcopy and Cervical Pathology members' opinion. J Obstet Gynaecol. 2018;38(3):38894.

22. McAllum B, Sykes PH, Sadler L, Macnab H, Simcock BJ, Mekhail AK. Is the treatment of CIN 2 always necessary in women under 25 years old? Am J Obstet Gynecol. 2011;205(5):478.e1-7.

23. Verguts J, Bronselaer B, Donders G, Arbyn M, Van Eldere J, Drijkoningen M, et al. Prediction of recurrence after treatment for high-grade cervical intraepithelial neoplasia: the role of human papillomavirus testing and age at conisation. BJOG. 2006;113(11):1303-7.

24. Acladious NN, Sutton C, Mandal D, Hopkins R, Zaklama M, Kitchener H. Persistant human papillomavirus infection and smoking increase risk of failure of treatment of cervical intraepithelial neoplasia (CIN). Int J Cancer. 2002;98(3):435-9.

25. Silver MI, Gage JC, Schiffman M, Fetterman B, Poitras NE, Lorey T, et al. Clinical Outcomes after Conservative Management of Cervical Intraepithelial Neoplasia Grade 2 (CIN2) in Women Ages 21-39 Years. Cancer Prev Res (Phila). 2018;11(3):165-70.

26. Godfrey MAL, Nikolopoulos M, Garner JE, Adib TR, Mukhopadhyay D, Rains JS, et al. Conservative management of cervical intraepithelial neoplasia grade 2 (CIN2) in women under 30 years of age: A cohort study. European Journal of Obstetrics \& Gynecology and Reproductive Biology. 2018;228:267-73.

27. Munro A, Powell RG, P AC, Bowen S, Spilsbury K, O'Leary P, et al. Spontaneous regression of CIN2 in women aged 18-24 years: a retrospective study of a state-wide population in Western Australia. Acta Obstet Gynecol Scand. 2016;95(3):291-8.

28. Loopik DL, Doucette S, Bekkers RL, Bentley JR. Regression and Progression Predictors of CIN2 in Women Younger Than 25 Years. J Low Genit Tract Dis. 2016;20(3):213-7.

29. Bleecker E, Koehler E, Smith J, Budwit D, Rahangdale L. Outcomes after management of young women with cervical intraepithelial neoplasia 2 with a 6 -month observation protocol. J Low Genit Tract Dis. 2014;18(1):46-9.

30. Park Y, Lee E, Choi J, Jeong S, Kim H-S. Comparison of the Abbott RealTime High-Risk Human Papillomavirus (HPV), Roche Cobas HPV, and Hybrid Capture 2 assays to direct sequencing and genotyping of HPV DNA. J Clin Microbiol. 2012;50(7):2359-65.

31. Darragh TM, Colgan TJ, Cox JT, Heller DS, Henry MR, Luff RD, et al. The Lower Anogenital Squamous Terminology Standardization Project for HPV-Associated Lesions: background and consensus recommendations from the College of American Pathologists and the American Society for Colposcopy and Cervical Pathology. Arch Pathol Lab Med. 2012;136(10):1266-97.

32. Lee MH, Finlayson SJ, Gukova K, Hanley G, Miller D, Sadownik LA. Outcomes of Conservative Management of High Grade Squamous Intraepithelial Lesions in Young Women. J Low Genit Tract Dis. 
2018;22(3):212-8.

33. Agramunt S, Checa MA, Gonzalez-Comadran M, Larrazabal F, Arbos A, Alameda F, et al. High-grade squamous intraepithelial lesion could be managed conservatively in women up to 25 years: results from a retrospective cohort study. J Low Genit Tract Dis. 2013;17(4):459-62.

34. Skorstengaard M, Lynge E, Suhr J, Napolitano G. Conservative management of women with cervical intraepithelial neoplasia grade 2 in Denmark: a cohort study. BJOG. 2019.

35. Arbyn M, Redman CWE, Verdoodt F, Kyrgiou M, Tzafetas M, Ghaem-Maghami S, et al. Incomplete excision of cervical precancer as a predictor of treatment failure: a systematic review and meta-analysis. Lancet Oncol. 2017;18(12):1665-79.

\section{Hosted file}

Figure 1 CONSORT diagram.pdf available at https://authorea.com/users/365158/articles/485374comparison-of-expectant-vs-excisional-ablative-management-for-cin2-in-the-era-of-hpvtesting

\section{Hosted file}

Table 1 Demographics.pdf available at https://authorea.com/users/365158/articles/485374comparison-of-expectant-vs-excisional-ablative-management-for-cin2-in-the-era-of-hpvtesting

\section{Hosted file}

Table 2 Risk factors and other characteristics in initial conservative management group.pdf available at https://authorea.com/users/365158/articles/485374-comparison-of-expectant-vsexcisional-ablative-management-for-cin2-in-the-era-of-hpv-testing

\section{Hosted file}

Table 3 Summary of characteristics of initial excision patients.pdf available at https: //authorea.com/users/365158/articles/485374-comparison-of-expectant-vs-excisionalablative-management-for-cin2-in-the-era-of-hpv-testing 